\title{
Formation of Quantum Dot Fluorescent Monolayer Film using Peptide Bond
}

\author{
Watau Inami $^{\dagger}$, Koichi Nanbu ${ }^{*}$, Atsuo Miyakawa*, and Yoshimasa Kawata* \\ (2011 년 10 월 2 일 접수; 2012 년 3 월 16 일 심사완료; 2012 년 3 월 23 일 게재확정)
}

\begin{abstract}
We present a method for preparing a quantum dot fluorescent monolayer film on a glass substrate. Since nanoparticles aggregate easily, it is difficult to prepare a nanoparticle monolayer film. We have used a covalent bond, the peptide bond, to fix quantum dots on the glass substrate. The surface of the quantum dot was functionalized with carboxyl groups, and the glass substrate was also functionalized with amino groups using a silane coupling agent. The carboxyl group can be strongly coupled to the amino group. We were able to successfully prepare a monolayer film of CdSe quantum dots on the glass substrate.
\end{abstract}

Key Words : Display, Fluorescent monolayer film, Peptide bond, Quantum dot

\section{Introduction}

Quantum dots are parts of matter whose excitons are confined in all three spatial dimensions. As a result, their electronic properties are between those of bulk semiconductors and those of discrete molecules. Research interest in quantum dots has tremendously increased in recent years because they are extremely promising materials that can be applied to various fields such as lasers ${ }^{1-3}$, light emitting $\operatorname{diodes}^{3-6}$, display ${ }^{7,8}$, transistors $^{9}$, fluorescent dyes for life science ${ }^{10,11}$, solar cells $^{12}$ and so on $^{13-15}$. Quantum dots have many advantages such as tunability of emission color with a single-excitation light source, wide color reproduction range, and long-term photostability. The emission wavelength of a quantum dot can by con- trolling its size: further the emission wavelength range is extremely wide and encompasses ultraviolet to near infrared (3501300nm).

A promising application of quantum dots is display devices, because the colors can be easily tuned by mixing several quantum dots and the emission can be excited by a single light source. A quantum dots thin film with a thickness of several tens of nanometers can realize

$\dagger$ Division of Global Research Leaders, Shizuoka University E-mail : dwinami@ipc.shizuoka.ac.jp

TEL : +81-(53)478-1371

* Faculty of Engineering, Shizuoka University ultra-thin flexible displays.

However, it is difficult to prepare a nanoparticle monolayer film because nanoparticles aggregate easily. Self-organization processes can solve the aggregation problem. The Langmuir-Blodgett (LB) technique ${ }^{16,17}$, convective assembly method ${ }^{18,19}$, electrophoresis ${ }^{20}$, and magnetrophoresis $^{21}$ are well-known methods of arranging of nanoparticles on a substrate. The nanoparticles can be easily removed from the substrate, because the nanoparticles and substrate are not bonded strongly with the self-organization process. The bonding is non-covalent bond, whose strength is considerably weaker than that of a covalent bond.

In this paper, we present a method for preparing quantum dot fluorescent monolayer films on surface of a glass substrate surface using covalent bonding. Peptide bonds ${ }^{22}$ are used to fix the quantum dots on the surface of the surfaces. The peptide bond is a covalent bond that is formed between carboxyl groups and amino groups by dehydration synthesis. The surfaces of the quantum dots were functionalized with carboxyl groups, and the glass substrate is functionalized with amino groups by a silane coupling agent. The reaction time and concentration of the quantum dot solution are also optimized. We were able to successfully prepare 10 - and 20 - nm quantum dot monolayer films on a glass substrate.

\section{Experiment}

Figure 1 shows our method for fixing quantum dots on a substrate by using the peptide bond. Amino groups are 


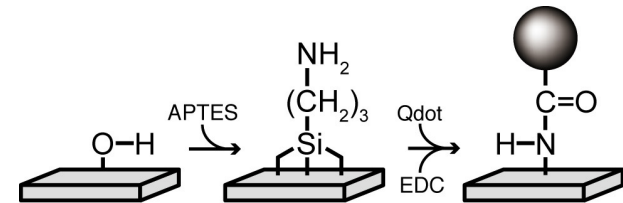

Fig.1 Schematic diagram of the method for obtaining quantum dot fluorescent monolayer film. Quantum dot and substrate are fixed by a peptide bond.

formed on the surface of a glass substrate by using a silane coupling agent. Quantum dots with polymer coating have carboxyl groups. The carboxyl groups of quantum dots react with the amino group of the surface glass substrate by dehydration synthesis, thereby releasing water. Peptide bonds are formed between the quantum dots and the glass substrate. The residual quantum dots that did not attach to the glass substrate can be removed by a rinsing process. In this manner, we can finally obtain a monolayer film of quantum dots.

Figure 2 shows our procedure for obtaining a quantum dot fluorescent monolayer film. The process consists of silanizing a glass surface, achieving coupling between quantum dots and the substrate, and rinsing procedure. First, amino groups were formed on a glass substrate surface by using a silane coupling agent. The glass substrate (Matsunami, C218181, 18x18mm) was then washed with Scat 20X PF (Daichi Clean Chemical,Inc.) detergent in an ultrasonic bath for $30 \mathrm{~min}$. Amino groups were formed on the surface of the glass substrate by immersing the glass film in a silane coupling agent for $5 \mathrm{~min}$. The silane coupling agent was prepared by mixing $200 \mu \mathrm{L}$ of 3 -aminopropyltriethoxy silane (APTES, Sigma, A3468) and 10mL of acetone (Wako, 018-17815). In order to wash the extra silane coupling agent from the glass substrate, the substrate was immersed in acetone in a plastic test tube and shaken. The substrate was then immersed in pure water to remove the acetone from the substrate surface. The rinsing process was performed twice.

The quantum dots that were functionalized with carboxyl groups were fixed on the surface of the glass substrate. A solution including the quantum dots was dropped on the glass substrate. The solution was prepared by adding $10.0 \mathrm{mg}$ of EDC (N - (3dimethylaminopropyl)- N $\quad$-ethylcarbodiimide hydrochloride(Sigma E6383)) to $1.0 \mathrm{~mL}$ of phosphate buffered saline (PBS; pH 7.4 (Sigma, P5368)). After EDC was completely dissolved in PBS, $10 \mu \mathrm{L}$ of a solution of quantum dots was added to the solution. The concentration of the prepared the quantum dot solution was $80 \mathrm{nM}$. We prepared a parafilm bank around the substrate to store the quantum dot solution on the substrate. The quantum dots were fixed on the substrate

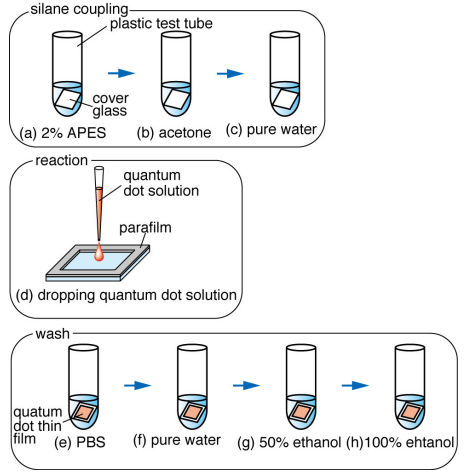

Fig.2 Procedure for preparing quantum dot fluorescent monolayer film.

by the formation of a peptide bond between the quantum dots and the substrate.

Finally, the glass substrate was washed in order to remove the residual the quantum dots from the substrate surface. The parafilm was removed and the glass substrate was immersed in PBS solution. Subsequently, the glass substrate was immersed in pure water. The rinsing procedure was performed twice. The glass substrate was successively immersed in $50 \%$ and $100 \%$ ethanol successively and dried at room temperature.

\section{Results and discussion}

Figure 3(a) shows the observation results of the fluorescent monolayer film of quantum dots. We used Qdot 655 ITKTM Carboxyl Quantum Dots (Invitrogen) prepared from nanometer-scale crystals of a semiconductor material $(\mathrm{CdSe})$. The diameter of the quantum dot we used was $20 \mathrm{~nm}$. We observed the topography of the monolayer film by using an atomic force microscope (AFM; Seiko Instruments, SPA300). The scanning area was $1 \times 1 \mu \mathrm{m}$. The reaction time and the concentration of the quantum dot solution were $1 \mathrm{~h}$ and 80nM, respectively. As shown in Fig. 3(a), a monolayer quantum dot film was formed with some particles having diameters larger than $20 \mathrm{~nm}$.

Figure 3(b) and (c) show the line profiles along lines (A) and (B), respectively, in Fig. 3(a). Figure 3(b) shows peaks of two different sizes: $21.9 \mathrm{~nm}$ and $54.1 \mathrm{~nm}$. The peak of $21.9 \mathrm{~nm}$ width represents a single quantum dot while the peak of the $54.1 \mathrm{~nm}$ width represents an aggregation of quantum dots. Some aggregations of quantum dots can be observed in Fig. 3(a). The height of the observed quantum dots in Fig. 3(b) and (c) was approximately $8 \mathrm{~nm}$ even though we had used quantum dots with a diameter of $20 \mathrm{~nm}$. We believe that this was because the quantum dot that formed on the substrate "sank" into amino groups whose thickness was $10 \mathrm{~nm}$. 


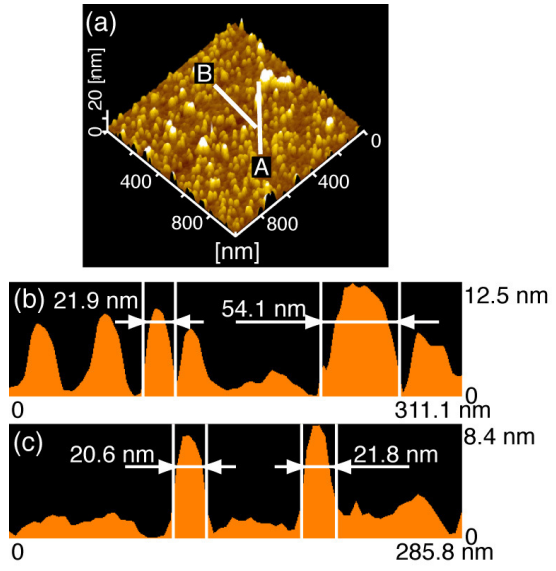

Fig.3 Observation results of quantum dot fluorescent monolayer film. The scanning area was $1 \times 1 \mu \mathrm{m}$, while the diameter of the quantum dot was $20 \mathrm{~nm}$. The reaction time was $1 \mathrm{~h}$ and the concentration of the quantum dot was $80 \mathrm{nM}$. (a) Atomic force microscope (AFM) image of quantum dot fluorescent monolayer film. (b) and (c) line profiles along lines $\mathrm{b}$ and $\mathrm{c}$ in (a).

EDC caused the excessive aggregations under the experimental conditions. Under the conditions, after EDC was added to the solution, the aggregation was occurred quickly and sank into the substrate. The EDC, which is a dehydration-condensation agent, causes a bond between the carboxyl and amino groups. Therefore, the aggregation did not occur, when a quantum dot solution was added to the PBS without the EDC. We believe that the aggregations occur due to excessive number of activated quantum dots. The amount of aggregations increases due to the activation of quantum dots by EDC. It is important to adjust the concentration of the quantum dot solution and the reaction time to reduce the probability of collisions of the quantum dots.

Figure 4 shows the AFM observation results of the quantum dot fluorescent monolayer films that were prepared by changing the reaction time and concentration of the quantum dot solution. The aggregations formed during the process were caused by adding EDC. In order to reduce the aggregations, the reaction time and the concentration of quantum dot solution were optimized and made shorter and lower, respectively: the reaction times were 5,10 , and $15 \mathrm{~min}$ and the concentration of the quantum dot solution was $8 \mathrm{nM}$. From the observation results, the quantum dots fluorescent monolayer film was uniform for all the reaction times. The coverage increased with longer reaction times. Some aggregations of the quantum dots were observed, but they did not increase with the reaction time.
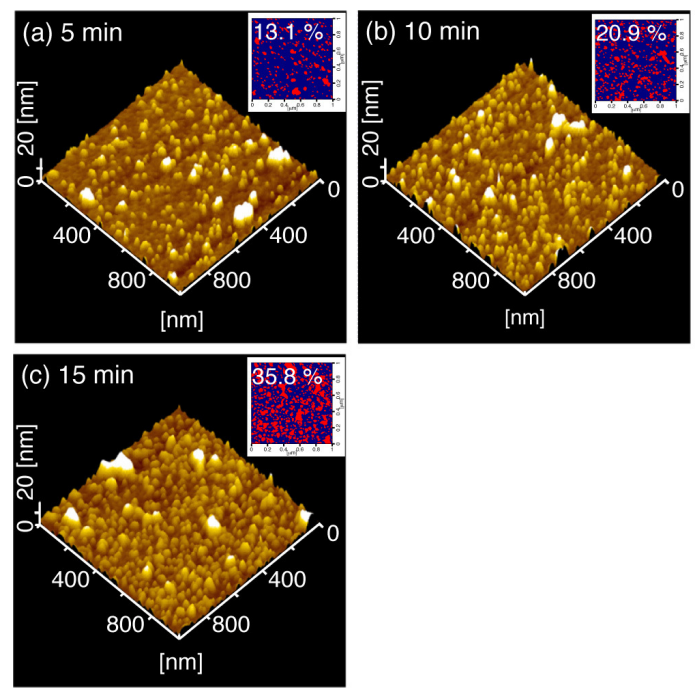

Fig.4 AFM observation results of the quantum dot fluorescent monolayer film. The reaction times were (a) 5, (b) 10, and (c)15min and the quantum dot concentration was $8 \mathrm{nM}$. Inset : Coverage of the quantum dot on the glass substrate as a function of reaction time.

The coverage of the quantum dots on the glass substrate as a function of the reaction time. The reaction time were (a) 5, (b) 10, and (c) $15 \mathrm{~min}$ and the coverages were $13.1 \%, 20.9 \%$, and $35.8 \%$, respectively. The coverage ratios increased with longer reaction times.

Figure 5(a) shows the schematic diagram of the experimental setup of the measurements of the emission light of the films. We used Qdot 525 ITK Carboxyl Quantum Dots and Qdot 655 ITK Carboxyl Quantum Dots (Invitorogen). Here, the sizes of quantum dots are 10 and $20 \mathrm{~nm}$, and the corresponding emission wavelengths are 525 and $655 \mathrm{~nm}$, respectively. The two films were piled and arranged in a zigzag order in Fig. 5(a). The stack boundaries of the quantum dot fluorescent monolayer films were observed by using a fluorescence microscope. The excitation wavelength was 360 - 370nm.

Figure 5(b) and (c) show the micrographs of the emission light from the quantum dot fluorescent monolayer films. In Fig. 5(b), the emission color is red on the left side and yellow on the right side. On the right side, both the quantum dots were excited simultaneously and emitted light at 525 and $655 \mathrm{~nm}$.

Therefore, we observed yellow color on the right side of Fig. 5(b). On the other hand, in Fig. 5(c), the emission color is green because the two films in this area did not overlap. The wavelengths of the emission also were also 525 and $655 \mathrm{~nm}$ on right side. The quantum dot fluorescent monolayer films and stacked quantum dot 
(a)
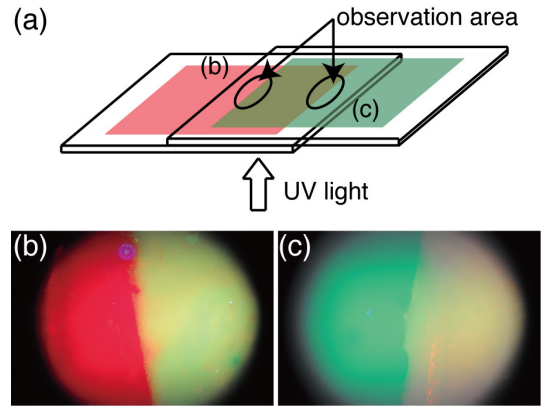

Fig.5 Light emission from the two quantum dot fluorescent monolayer films excited by a single light source. 10- and 20-nm diameter quantum dots were used. The emission wavelengths are 525 and $655 \mathrm{~nm}$, respectively. (a) schematic diagram of experimental setup of excitation of the films. (b) and (c) photographs of emission light from quantum dot fluorescent monolayer films.

fluorescent films can be excited by a single light source, and the emission color can be finely controlled by stacking the fluorescent films.

\section{Conclusion}

In this study, we have demonstrated the method for fabricating a quantum dot fluorescent monolayer film on a glass substrate and have succeeded in preparing 10and 20-nm quantum dot monolayer films by using a peptide bond on a glass substrate. We optimized the reaction time and concentration of quantum dots. As a result, a highly uniform quantum dot fluorescent monolayer film was obtained because the aggregation of the quantum dots could be reduced. The density of the quantum dots on the monolayer film could be controlled by varying two key factors - reaction time and concentration of quantum dot solution. The quantum dots are strongly attached to the substrate because the peptide bond between the quantum dot and substrate is strong and rigid. Yellow color was synthesized by overlapping of two fluorescent monolayer films having emission wavelengths were 525 and $655 \mathrm{~nm}$, and the two films were excited simultaneously by a single light source.

It is possible to contruct pixelized quantum dots on a substrate using photolithography techniques and arrange various quantum dots on one substrate. The method can also be applied to flexible films such as polyimide and polyethylene terephthalate films. Since the quantum dots are attached to the film with a covalent bond, they may not become detached if a flexible film is bent. We believe that our method can be widely applied to various photonics fields such as displays, illuminations, solar cells, biosensors, and memory devices.

\section{Acknowledgement}

This work has been supported by CREST of JST (Japan Science and Technology Agency).

\section{Reference}

[1] V. I. Klimov, A. A. Mikhailovsky, S. Xu, A. Malko, J. A. Hollingsworth, C. A. Leatherdale, H. J. Eisler, and M. G. Bawendi, 2000, "Optical gain and stimulated emission in nanocrystal quantum dots," Science 290, pp.314-317.

[2] D. L. Huffaker, G. Park, Z. Zou, O. B. Shchekin, and D. G. Deppe, 1998, “1.3 $\mu \mathrm{m}$ room- temperature GaAs-based quantum-dot laser," Appl. Phys. Lett. 73, pp.2564-2566.

[3] A. J. Shields, "Semiconductor Quantum Light Sources," Nature Photonics 1 pp.215-223 (2007).

[4] M. C. Schlamp, X. G. Peng, and A. P. Alivisatos, 1997, "Improved efficiencies in light emitting diodes made with $\mathrm{CdSe}(\mathrm{CdS})$ core/shell type nanocrystals and a semiconducting polymer," J. Appl. Phys. 82, pp.5837-5842.

[5] N. M. Park, T. S. Kim, and S. J. Park, 2001, "Band gap engineering of amorphous silicon quantum dots for light-emitting diodes," Appl. Phys. Lett. 78, pp.2575-2577.

[6] S. Coe, W. K. Woo, M. Bawendi, and V. Bulovic, 2002, "Electroluminescence from single monolayers of nanocrystals in molecular organic devices," Nature 420, pp.800-803.

[7] T. H. Kim, K. S. Cho, E. K. Lee, S. J. Lee, J. Chae, J. W. Kim, D H. Kim, J. Y. Kwon, G. Amaratunga, S. Y. Lee, B. L. Choi, Y. Kuk, J. M. Kim, and K. Kim, 2011, "Full-colour quantum dot displays fabricated by transfer printing," Nature Photonics 5, pp.176-182.

[8] E. Jang, S. Jun, H. Jang, J. Lim, B. Kim, and Y. Kim, 2010, "White-Light-Emitting Diodes with Quantum Dot Color Converters for Display Backlights," Adv Mater. 22, pp.3076-3080.

[9] H. Park, J. Park, A. K. L. Lim, E. H. Anderson, A. P. Alivisatos, and P. L. McEuen, 2000, "Nanomechanical oscillations in a single- $\mathrm{C}_{60}$ transistor," Nature 407, pp.57-60.

[10] C. W. Warren Chan, and S. Nie, 1998, "Quantum Dot Bioconjugates for Ultrasensitive Nonisotopic Detection," Science 281, pp.2016-2018.

[11] I. L. Medintz, H. T. Uyeda, E. R. Goldman, and H. Mattoussi, 2005, "Quantum dot bioconjugates for imaging, labelling and sensing," Nature Materials 4, pp.435-446. 
[12] S. Robel, V. Subramanian, M. Kuno, and P. V. Kamat, 2006, "Quantum Dot Solar Cells. Harvesting Light Energy with CdSe Nanocrystals Molecularly Linked to Mesoscopic TiO2 Films," J. Am. Chem. Soc. 128, pp.2385-2393.

[13] N. H. Bonadeo, J. Erland, D. Gammon, D. Park, D. S. Katzer, and D. G. Steel, 1998, "Coherent Optical Control of the Quantum State of a Single Quantum Dot," Science 282, pp.1473-1476.

[14] A. Imamoglu, D. D. Awschalom, G. Burkard, D. P. DiVincenzo, D. Loss, M. Sherwin, and A. Small, 1999, "Quantum Information Processing Using Quantum Dot Spins and Cavity QED," Phys. Rev. Lett. 83, pp.4204-4207.

[15] P. Michler, A. Kiraz, C. Becher, W. V. Schoenfeld, P. M. Petroff, L. Zhang, E. Hu, and A. Imamoglu, 2000, “A Quantum Dot Single-Photon Turnstile Device," Science 290, pp.2282-2285.

[16] F. Lenzmann, K. Li, A. H. Kitai, and H. D. H. Stover, 1994, "Thin-film micropatterning using polymer microspheres," Chem. Mater. 6, pp.156-159.

[17] K. U. Fulda, and B. Tieke, 1994, “Langmuir films of monodisperse $0.5 \mu \mathrm{m}$ spherical polymer particles with a hydrophobic core and a hydrophilic shell," Adv. Mater. 6, pp.288-290.

[18] N. D. Denkov, O. D. Velev, P. A. Kralchevsky, I. B. Ivanov, H. Yoshimura, and K. Nagayama, 1993, "Two-dimensional crystallization," Nature 361, pp.2626.

[19] A. S. Dimitrov, and K. Nagayama, 1996, "Continuous Convective Assembling of Fine Particles into Two-Dimensional Arrays on Solid Surfaces," Langmuir 12, pp.1303-1311.

[20] E. Sabatani, and I. Rubinstein, 1987, “Organized self-assembling monolayers on electrodes. 2. Monolayer-based ultramicroelectrodes for the study of very rapid electrode kinetics," J. Phys. Chem. 91, pp.6663-6669.

[21] C. Kermel, V. Lardot, D. Libert, and I. Urbain, 2001, "Grain Oriented Microstructure Made in High Magnetic Field," Key Eng. Mater. 206-213, pp.445448.

[22] L. Pauling, 1960, The Nature of the Chemical Bond and the Structure of Molecules and Crystals; An Introduction to Modern Structural Chemistry (Cornell Univ. Press, Ithica, NY). 